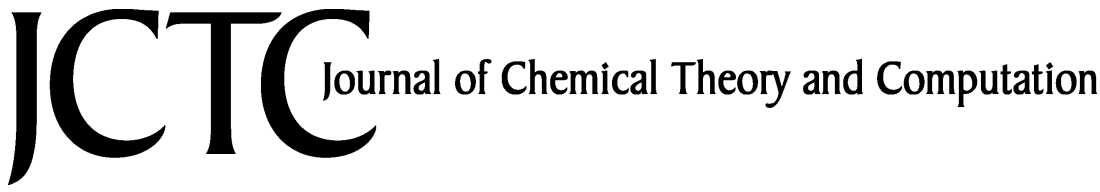

\section{Dependence of Spurious Charge-Transfer Excited States on Orbital Exchange in TDDFT: Large Molecules and Clusters}

\author{
R. J. Magyar \\ NIST Center for Theoretical and Computational Nanosciences (NCTCN), \\ Gaithersburg, Maryland 20899, and Theoretical Division, Los Alamos National \\ Laboratory, Los Alamos, New Mexico 87545 \\ S. Tretiak* \\ Theoretical Division and Center for Nonlinear Studies, Los Alamos National \\ Laboratory, Los Alamos, New Mexico 87545
}

Received September 9, 2006

\begin{abstract}
Time-dependent density functional theory (TDDFT) is a powerful tool allowing for accurate description of excited states in many nanoscale molecular systems; however, its application to large molecules may be plagued with difficulties that are not immediately obvious from previous experiences of applying TDDFT to small molecules. In TDDFT, the appearance of spurious charge-transfer states below the first optical excited state is shown to have significant effects on the predicted absorption and emission spectra of several donor-acceptor substituted molecules. The same problem affects the predictions of electronic spectra of molecular aggregates formed from weakly interacting chromophores. For selected benchmark cases, we show that today's popular density functionals, such as purely local (Local Density Approximation, LDA) and semilocal (Generalized Gradient Approximation, GGA) models, are qualitatively wrong. Nonlocal hybrid approximations including both semiempirical (B3LYP) and ab initio (PBE1PBE) containing a small fraction (20-25\%) of Fock-like orbital exchange are also susceptible to such problems. Functionals that contain a larger fraction $(50 \%)$ of orbital exchange like the early hybrid (BHandHLYP) are shown to exhibit far fewer spurious charge-transfer (CT) states at the expense of accuracy. Based on the trends observed in this study and our previous experience we formulate several practical approaches to overcome these difficulties providing a reliable description of electronic excitations in nanosystems.
\end{abstract}

\section{Introduction}

Density functional theory (DFT) is one of the most promising tools to provide an accurate yet computationally feasible electronic-structure theory for large nanoscale systems, and the time-dependent extension of DFT (TDDFT) makes possible perhaps the only first principles approach to access

\footnotetext{
* Corresponding author e-mail: serg@lanl.gov.
}

the optical properties of large molecular systems containing hundreds of atoms. ${ }^{1-3}$ Combined the two have been successful in determining ground-state geometries, vibrational spectra, and photoexcitations of many small molecules. ${ }^{4,5}$ These days, accurate measurements are now readily available through various absorption and emission time- and frequencyresolved spectroscopies even for large molecular systems and their assemblies. The understanding and controlled manipulation of optical properties of nanoscale molecular clusters 
may lead to many technological applications such as lightharvesting and photovoltaic devices ${ }^{6,7}$ and precision chemoand biosensors ${ }^{8,9}$ to name just a few. Several comprehensive reviews of quantum-chemical methodology for UV-visible spectra of large molecules has been published recently (e.g. refs 10 and 11). So there is a clear need to apply TDDFT here.

Density functional theory formally permits the expression of the total ground-state energy and other properties of a quantum many-body system as functionals of the electron density alone and provides a formally exact scheme for solving the many-body problem. ${ }^{12}$ TDDFT is an extension of density functional theory in which many-body excitations are associated with the poles of the exact density response. ${ }^{3,13,14}$ Practical density functionals are approximate and typically make use of an auxiliary orbital-based KohnSham (KS) scheme in which the kinetic energy contribution of the total energy is expressed as an orbital functional. The only contribution to the total energy that must be approximated is the exchange-correlation contribution. This contribution is often approximated by functionals which depend on the electronic density locally (Local Density Approximation, LDA) or semilocally (Generalized Gradient Approximation, GGA) in a way convenient for calculations. However, more accurate functionals require further longrange information about the density which is contained in the Kohn-Sham orbitals. A step toward a general orbital exchange-correlation functional is the exact-exchange (EXX) which has recently become popular in the solid state-physics community. Results within EXX should exhibit both the proper asymptotic behavior for the effective potential, $\sim 1 / r$ for finite systems, and a derivative discontinuity as the number of particles changes through integer values. Properly describing these could mean more accurate band-gaps and optical spectra. We note too that long-range effects will become important when treating the excited states of the jellium model. These problems are discussed in detail in refs 14-16.

Orbital exchange in the DFT formalism is the exact orbital contribution of exchange to the exchange-correlation functional and can be written as a Fock-like integral over the KS orbitals. ${ }^{17,18}$ The rewards for using orbital exchange are tempting and include more realistic potentials, better decaying KS orbitals, and more accurate excited states. However, extracting the local potential and its derivative kernel from this exact-exchange functional is difficult and in practice, is often done approximately by replacing the local exactexchange potential by a nonlocal Hartree-Fock exchange. All current routine applications of TDDFT are based on the adiabatic approximation, where the memory effects in the time-dependent density evolution are neglected. ${ }^{19,20}$ The adiabatic approximation, for example, makes it impossible to describe highly correlated electronic states such as those having predominantly double character. ${ }^{21}$ It has been argued that omission of nonadiabatic effects is also solely responsible for the charge-transfer problems. ${ }^{22}$ In this paper, we show that long-range orbital-based exchange improves upon the semilocal approximations when describing charge-transfer states. This can be rationalized by noting that the hybrid
TDDFT kernel constitutes a specific case of nonadiabatic approximation. ${ }^{23}$

One of the greatest challenges to the universal applicability of practical TDDFT is the inability of popular density functionals (and their kernels) to routinely and accurately describe certain excitations with a long-range spatial extent. ${ }^{24-27}$ For example, charge-transfer (CT) states correspond to excited states in which the photoexcited hole and electron do not greatly overlap due to incorrect long-range functional asymptotics and the missing discontinuity of the approximate xc-potentials with respect to particle number. Subsequently, TDDFT often predicts charge-transfer states of substantially lower energy and below optical states. A heuristic way to understand this is to consider the KS orbital excitations as zeroth-order approximations to the true excitations of the system. The TDDFT linear response formalism can be thought of as the inclusion of an effective mixing determined by the exchange-correlation kernel between the pair of occupied and unoccupied KS orbitals that constitutes the excited state. With the most commonly used approximate kernels, such as LDA and GGA models, many bound exciton states are not described at all. ${ }^{27,28}$ This is because the kernal is local and the orbital overlap is negligible. This problem is further complicated by the neglect of an important derivative discontinuity in most functionals. This results in a mismatch of ionization potentials between the donor and acceptor portions of molecule. ${ }^{29}$ Thus, the energies of CT states are frequently so significantly lower than what they should be that these states often become the lowest energy states in calculated electronic spectra.

These TDDFT shortcomings have been well identified and explored in the case of small molecular systems and local approximations. In this work, we explore how DFT's predictive power changes when we simulate the ground and excited states of larger molecules and aggregates. In particular, we investigate the appearance of low-lying unphysical CT states which depends on the choice of density functional. We first focus on ground- and excited-state geometries in the substituted molecules. Such calculations constitute a common approach to investigate the coupling of electronic and vibrational degrees of freedom (frequently quantified as Frank-Condon overlaps) and to calculate spectroscopically observed vibrational structure in absorption and fluorescence spectral line shapes. ${ }^{30,31} \mathrm{We}$ next study electronic interactions mediated by aggregation in molecular assemblies. Computation of small molecular aggregates is a typical technique to determine interchromophore interactions in molecular assemblies. ${ }^{32-34}$ This allows determination of aggregation type $(\mathrm{H}$ or $\mathrm{J})$ and electronic coupling energies. The calculated information is useful for building reduced Frenkel exciton models, for example, one for Light-Harvesting Complex II (LHCII). ${ }^{35,36}$ Such tasks extend well beyond a standard simple case of ground-state geometry optimizations followed by excited-state calculations and constitute the area where the performance of TDDFT has not been consistently tested yet.

We chose representative molecules with properties that could be useful for several important technological applications: donor-donor and donor-acceptor substituted molecules and aggregates of thiophene oligomers and bacteri- 


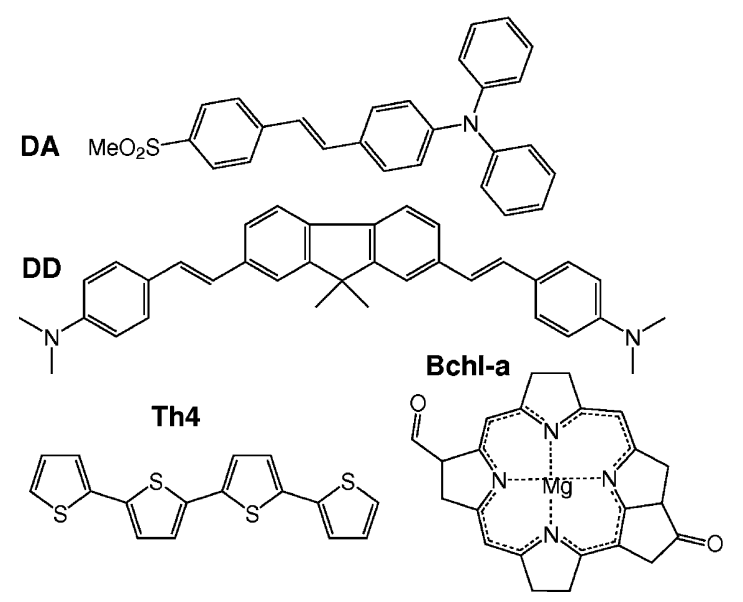

Figure 1. Chemical diagrams of the molecules studied: donor-acceptor (DA) and donor-donor (DD) substituted chromophores, thiophene oligomer (Th4), and bacteriochlorophyll a (Bchl-a).

ochlorophylls. Substituted organic chromophores are promising candidates in several areas, such as nonlinear optical response applications. ${ }^{37,38}$ The thiophenes have been exploited for applications in organic electronics related to novel display, photovoltaic, and lighting technologies. ${ }^{39,40}$ Finally, the chlorophylls constitute the main pigment in the biological photosynthetic light-harvesting complexes. ${ }^{35,36}$ Previous theoretical work within DFT on some of these systems has been carried out within the DFT formalism for donor-acceptor molecules, ${ }^{41,42}$ thiophenes, ${ }^{43}$ and bacteriochlorophylls. ${ }^{44,45}$ Here our calculations examine the qualitative and quantitative aspects of TDDFT for excited-state molecular electronic structures. The results are compared for several commonly used functionals with varying fractions of Hartree-Fock (HF) exchange as implemented in standard quantum chemical packages. By tuning the fraction of HF exchange we hope to gain insight into how the orbital dependence of the exchange function affects the description of electronic excited states.

Details of our numerical modeling are presented in section II. In section III we analyze computational results obtained via different DFT approximations. Finally, we discuss the trends that emerge and summarize our findings in section IV.

\section{Computational Methodology}

Figure 1 shows the chemical structures of the molecules considered, including donor-acceptor DA ((E)-4-(4-(methylsulfonyl)styryl)-N,N-diphenylbenzenamine) and donordonor DD (4,4'-[(1E,1'E)-2,2'-(biphenyl-4,4'-diyl) bis(ethene$1,1^{\prime}$-diyl)]bis(N,N-dimethylbenzenamine)) substituted molecules, thiophene oligomer (Th4), and bacteriochlorophyll- $a$ (Bchl-a).

Ground-state geometry optimizations of the charge-neutral, singlet states of the substituted molecules have been done with the Turbomole program suite. ${ }^{46}$ The lowest excitedstate geometries were obtained next using Turbomole as well. This code is able to search for a minimum over the TDDFT excitation energy surface with respect to nuclear coordinates using analytic gradient techniques. ${ }^{47}$ All optimizations were performed in the gas phase using the SV basis set and one of a set of density functionals; see below. The SV and similar 6-31G basis sets are known to be an efficient blend of accuracy and manageable size for large conjugated molecules. ${ }^{48}$ For the thiophenes and chlorophyll calculations we used geometries extracted from the experimental X-ray crystallographic data. ${ }^{49,50}$ In addition to the single molecule limit, we consider thiophene dimers (2Th4) generated from the coordinates of two nearest neighbor pairs in the unit cell. ${ }^{49}$ For the single molecules, two Th4 geometries exist in the crystal structure. One structure is slightly more elongated. The calculated excited-state spectra are essentially identical for both geometries. Similarly, the coordinates of $\alpha \beta$ Bchls- $a$ pair (2Bchl-a) have been extracted from Rs. molischianum crystal data. ${ }^{50}$ At the obtained geometries we calculate corresponding excited-state structures up to 20 lowest excited states using the Gaussian 03 package ${ }^{51}$ with a $6-31 \mathrm{G}$ basis set and density functionals corresponding to the methods used for geometry optimizations (unless specified otherwise).

We use several common density functionals with various fractions of orbital exchange, namely HF (100\%), BHandHLYP (50\%), PBE1PBE (25\%), B3LYP (20\%), TPSS-H (10\%), TPSS (0\%), BP86 (0\%), and SVWN (0\%). The set represents a gradual decreasing fraction of exchange. The treatments of correlation in each functional are not comparable and do not scale with the given fractions of exchange; however, the effects of correlation are assumed to be smaller than the relative error in the exchange amount. General trends seen as a function of the fraction of orbital exchange should persist regardless of the details of the correlation functional. We expect that functionals, including exchange, are less dramatically affected by CT problems. However, when the faction of exchange becomes large, these functionals typically provide less accurate energetics because the cancellation of errors between exchange and correlation is reduced. ${ }^{52}$ For example, the BHandHLYP functional ${ }^{53}$ combines semilocal exchange-correlation with orbital exchange in a 50-50 ratio. By construction, this functional handles a large fraction of long-range exchange exactly but fails to describe correlation in a compatible way.

To interpret computational trends we use a transition orbital analysis, which allows precise identification and visualization of the electronic excitations in question. ${ }^{54}$ These transition orbitals provide a graphical real-space representation of the transition densities associated with the molecular electronic excitations computed with TDDFT. This analysis offers the most compact description of a given transition density in terms of its expansion in single $\mathrm{KS}$ transitions. The Gaussian 03 code was locally modified to be able to perform the transition orbital analysis.

\section{Results and Discussion}

Through a series of benchmark calculations, we highlight the difficulties appearing when we apply TDDFT to molecular systems of sizes important to nanoscale applications. An important issue with using TDDFT for larger molecular systems is the introduction of spurious low-energy CT states. In the following subsections, we investigate several fre- 


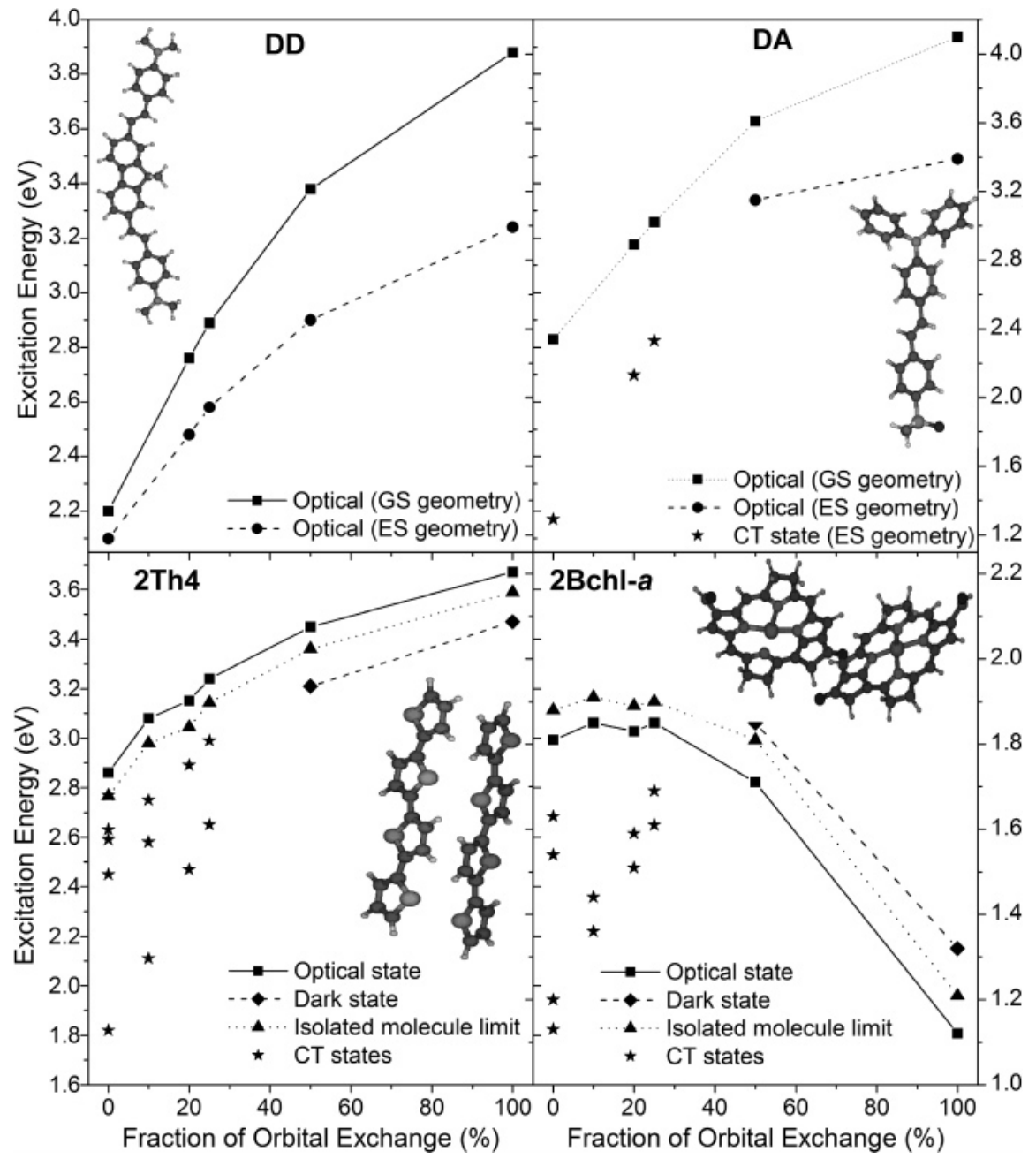

Figure 2. The calculated optical excitation energy as a function of the percentage of orbital exchange for the ground-state (GS) and the first excited-state (ES) optimized geometries of the donor-donor (DD) and donor-acceptor (DA) molecules just the GS for the $\mathbf{2 T h} 4$ and $\mathbf{2 B c h l}-\mathbf{a}$ dimers.

quently unexpected manifestations of this problem and explore their implications.

We arbitrarily label three types of singlet excited states: 'optical', 'dark', and 'charge transfer'. The optical excitation has a nonvanishing oscillator-strength, which means that it is possible to detect this excited state spectroscopically. The dark state refers to a low-energy electronic excitation with vanishing oscillator strength, that will not appear in the optical absorption. A CT state is a specific case of 'dark' state with a highly ionic nature where the photoexcited electron and hole have significant spatial separation. The oscillator strengths of CT states are expected to be weak because the overlap between the charge-transfer state and the ground state is extremely small and so are the transition dipole moments between these states. For example, in organic molecular crystals, CT states are usually higher in energy than the relevant Frenkel-exciton band of optical and dark states. ${ }^{55}$ However, DFT methods drastically underestimate the energies of these states. For this reason, all three types of states in dimers might be mixed, and their clear distinction is not possible. The CT states become particularly troublesome in the optimization of the excited-state geometries when the target excited state is not clearly constrained by symmetry, and level crossings may occur.
A. Donor-Acceptor Substituted Chromophores. We begin this investigation by examining a relatively large (approximately 70 atoms) molecular donor-donor (DD) system. This molecule has a long $\pi$-conjugated backbone and donors (etholanimes) at both ends of the backbone. This is perhaps the easiest test case for TDDFT and offers the best opportunity for the theory to provide accurate results. The molecule has no strong electron attractors, and we do not expect large-scale charge transfer with electrons and holes migrating from/to opposite ends of the molecule. The charge transfer should be of a mild nature from the donors to the conjugated backbone. In the upper left-hand corner of Figure 2 , we plot the energy of the lowest excited state versus functional for the DD molecule at both ground- and excitedstate optimal geometries. The respective calculated excitation energies and their oscillator strengths are listed in Table 1. The experimental values are from refs 42 and 56. Overall, functionals with a moderate fraction of orbital exchange such as B3LYP provide the best agreement with the experimental absorption and fluorescence maxima, neglecting the proper treatment of vibronic, temperature, and nonpolar solvent effects.

The lowest-energy (band gap) transition in such conjugated molecules typically has a remarkable oscillator strength and 
Table 1. Calculated Excitation Energies (eV) of the Donor-Donor (DD) and Donor-Acceptor (DA) Compounds ${ }^{a}$

\begin{tabular}{clrcl}
\hline molecule & method & \% exchange & GS & ES \\
\hline DD & HF & 100 & $3.88(2.56)$ & $3.24(2.58)$ \\
DD & BHandHLYP & 50 & $3.38(2.72)$ & $2.90(2.80)$ \\
DD & PBE1PBE & 25 & $2.89(2.43)$ & $2.58(2.63)$ \\
DD & B3LYP & 20 & $2.76(2.29)$ & $2.48(2.53)$ \\
DD & BP86 & 0 & $2.20(1.61)$ & $2.1(1.2)$ \\
DA & HF & 100 & $4.27(1.57)$ & $3.39(1.64)$ \\
DA & BHandHLYP & 50 & $3.61(1.40)$ & $3.15(1.50)$ \\
DA & PBE1PBE & 25 & $3.02(1.06)$ & $2.33(0.13)$ \\
DA & B3LYP & 20 & $2.89(0.99)$ & $2.13(0.08)$ \\
DA & BP86 & 0 & $2.34(0.72)$ & $1.29(0.02)$ \\
\hline
\end{tabular}

a The corresponding oscillator strengths $f$ are given in parentheses. GS and ES refer to the ground-state and the first excited-state optimized molecular geometries, respectively. The SV basis set was used for all calculations. Experimental absorption and fluorescence maxima are $\omega_{\text {abs }}=3.0 \mathrm{eV}$ and $\omega_{\mathrm{fl}}=2.7 \mathrm{eV}$ for $\mathrm{DD}$ and $\omega_{\mathrm{abs}}=3.2$ $\mathrm{eV}$ and $\omega_{\mathrm{fl}}=2.7 \mathrm{eV}$ for DA.

characteristically appears as a main peak in the linear absorption spectra. ${ }^{41,42}$ The calculated excited-state energy shifts to the blue with an increase of the fraction of orbital exchange. Likewise, the oscillator strength of the transition follows the fraction of exchange. These trends are expected since exact exchange cancels Coulomb self-interaction, and, thus, a larger fraction of orbital exchange corresponds to a contracted core. The valence states are also more tightly bound by the orbital exchange potential, but the effect is less extreme compared to the core contraction, since the HF virtual orbitals are calculated for the $\mathrm{N}+1$ electron system and their energies correspond to electron affinities rather than excitation energies. Consequently, the KS orbitals become widely separated. This is consistent with results seen from noble-gas solids and atoms treated by exact-exchange DFT. ${ }^{57}$ Since HF has even more weakly bound virtual orbitals than EXX, we expect the effect to be somewhat overemphasized. The energy of the first singlet state calculated at a relaxed excited-state geometry exhibits similar trends as a function of the fraction of orbital exchange. During excited-state geometry optimization the bonds along the conjugated backbone stretch, so that the bond-length alternation caused by uneven distribution of $\pi$-electronic density reduces. ${ }^{28}$ This allows the electrons to become more delocalized, and the excited-state energy is reduced. The magnitude of the observed Stokes shift and the respective geometry changes intensify with an increase of the fraction of exchange.

To analyze the nature of the discussed excited state, Table 2 displays the transition orbitals of the DD molecule obtained at the HF and BP86 limits, respectively. The two represent extremes in the locality of the functional. BP86 has only semilocal density-based exchange, while HF relies on the full orbital exchange. For the DD molecule, we see no qualitative difference between the HF and BP86 transition orbitals: they represent delocalized electronic state with electronic density slightly shifting toward the middle of the molecule upon photoexcitation. Transition orbitals describing emitting states are qualitatively similar to that of the absorbing state. We note that the BP86 orbitals are slightly more delocalized compared to the HF ones. This is due to the short-range description embodied in the BP86 functional. However, there is a quantitative difference in the energetics. This is consistent with the well-known fact that local density functionals are known to perform poorly for certain properties in the limit of longer conjugated chains. ${ }^{28}$ The conjugation length in this donor-donor (DD) molecule is perhaps too short for these semilocal functional shortcomings to become visually dramatic, but they remain energetically significant.

A more difficult test case is the donor-acceptor (DA) molecule in which the charge transfer between the donor and acceptor regions is energetically favorable and pronounced. It is hard to properly describe the underlying longrange interactions behind CT using a semilocal functional. The upper right-hand corner of Figure 2 shows scaling of the lowest singlet-state energy for the DA molecule, and the calculated data are presented in Table 1. As for the DD molecule, the excitation energy calculated at the groundstate geometry dramatically increases with the increasing fraction of exchange. This state is optically allowed having substantial oscillator strength. The HF transition orbitals shown in Table 2 reveal this excitation of $\pi-\pi$ nature delocalized between donor and acceptor. Steric distortion prevents participation of terminal phenyls, and the state has only weak CT character. In contrast, BP86 transition orbitals display strong CT character in this excited state (see Table 2). Since the transition is delocalized, the overlap between electron and hole wave-functions is small, which results in a reduced oscillator strength compared to the other methods. BP86 shows to the incorrect physical behavior of semilocal functionals. The accurate numbers should lie somewhere in between HF and BP86 extremes, and this is where the hybrid TDDFT excels. ${ }^{42}$ The situation with semilocal functionals becomes even more problematic after excitedstate optimization. While the functionals with a large percentage of orbital exchange (HF and BHandHLYP) behave normally, other methods converge to low-lying spurious CT states, where spatial overlap between an electron and a hole orbital is negligible (see Table 2), making the excitation optically forbidden (Table 1). A significant rearrangement of the molecular geometry is observed during excited-state geometry optimization, and this facilitates the crossover to the ionic CT state with a huge $1 \mathrm{eV}$ Stokes shift.

To rationalize that such ionic states are artifacts of the method, we recall that the ground- and lowest-energy excited electronic states of substituted push-pull molecules are often described as a combination of neutral and zwitterionic basis states represented by the corresponding molecular resonance forms. ${ }^{58,59}$ The zwitterionic state assumes full separation of positive and negative charges, and, consequently, it is optically forbidden. The excited state usually possesses greater zwitterionic basis state character than the ground state. The molecular structures also become more zwitterionic in character as solvent polarity increases. ${ }^{59}$ The semilocal functionals and functionals with a small fraction of orbital exchange do predict the ground state to be the mixture of such states; however, the excited state is described to be of solely zwitterionic character (see Table 2). In contrast, the DA molecule exhibits pronounced fluorescence properties, ${ }^{42}$ 
Table 2. Hartree-Fock and BP86 Transition Orbitals of the Lowest Excited State for the Ground-State (GS) Geometries of the Donor-Donor (DD) and Donor-Acceptor (DA) Molecules ${ }^{a}$

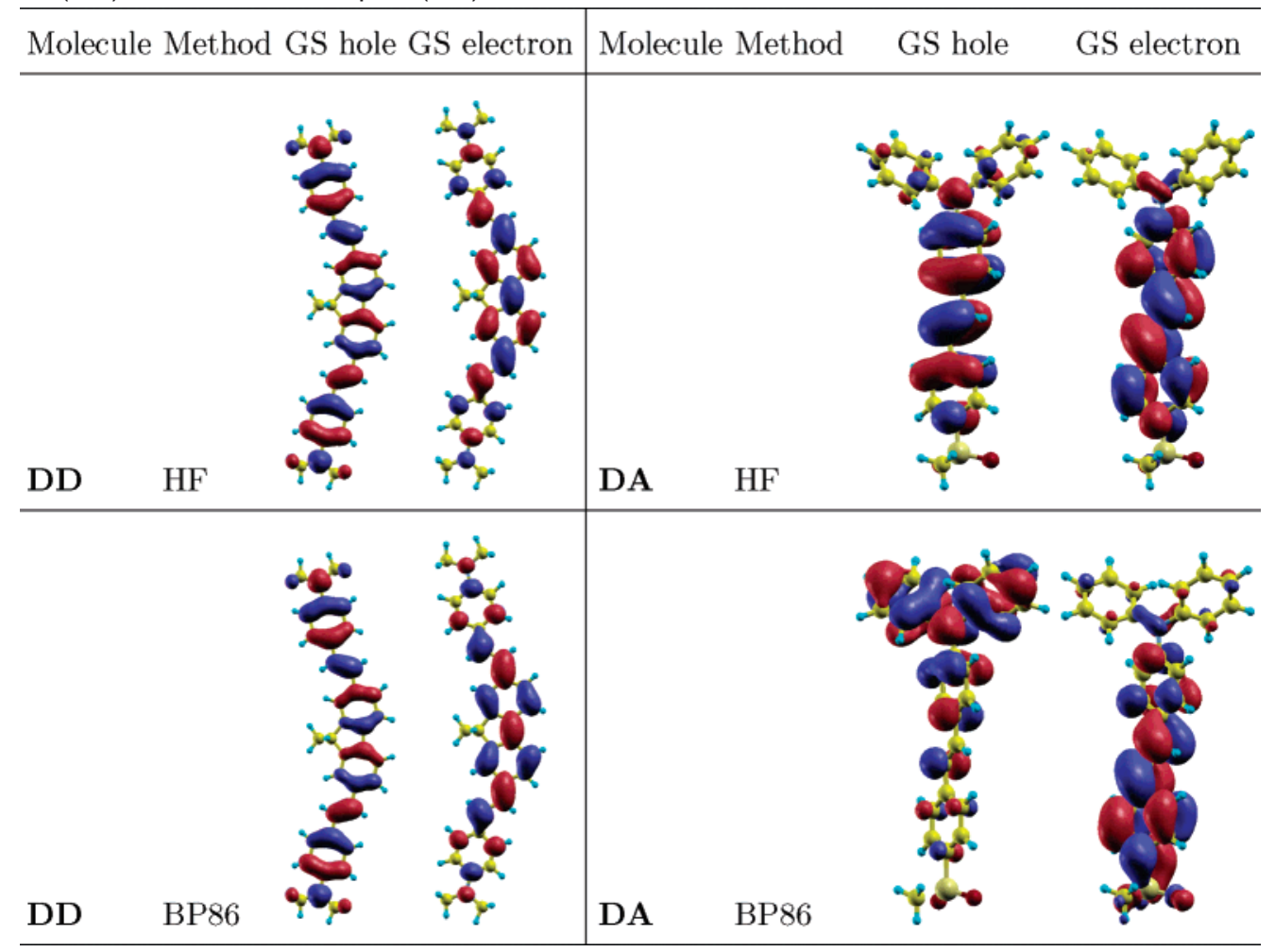

${ }^{a}$ These orbitals represent the full orbital exchange and semilocal functional limits, respectively. GS refers to the ground-state optimized molecular geometry.

pointing to the strong component of the neutral basis in the excited state. The observed emission stems from the lowest vibronic state, and Kasha's rule is not violated. Thus all available experimental data point that the DA compound does not exhibit dual-fluorescence properties as observed on other molecular systems such as 4-(dimethyl)aminobenzonitrile (DMABN) ${ }^{60}$ Moreover, computational excited-state geometry optimizations of the bright and the CT state for selected GGA functionals (not shown) do not change the relative state ordering. An opposite extreme is observed at the HF level when both ground and excited states have a dominant neutral component. Subsequently, the final result has a strong dependence on the fraction of orbital exchange used in the functional, which lessens the predictive power of TDDFT in applications to the polar substituted molecules.

Finally, in order to assess the basis set dependence, we performed several single point calculations on the DA molecule with split-valence basis sets of increasing complexity. The simplest is the $6-31 \mathrm{G}$ basis, then the same with polarization functions $\left(6-31 \mathrm{G}^{*}\right)$, then with diffuse functions $(6-31+G)$, and with both $\left(6-31+G^{*}\right)$. Table 3 shows the lowest optical excitation energy for both the original Turbomole SV-optimized geometry obtained for a given functional in vacuo and then the native geometry. The latter is the optimized structure consistent with the level of theory and basis set used for excited-state calculations. Adding diffuse functions alone shifted the vertical absorption spectrum by a maximum of $0.13 \mathrm{eV}$ to the red, and adding polarization functions shifted the spectrum by $0.1 \mathrm{eV}$ to the red as well with the most dramatic basis set effect for pure HF. These changes are less dramatic when native geometry is used. In practice, these molecules are often studied in solution, so we estimate solvent effects using the Polarizable Continuum Model (PCM) based on the Integral Equation Formalism $^{61-64}$ for toluene. Adding PCM stabilized the vertical absorption maximum by $0.15 \mathrm{eV}$ to the red with the most dramatic effects for the BP86 GGA kernel (see Table 3). Subsequently, using extended basis sets and solvent models leads to the overall $0.1-0.25 \mathrm{eV}$ red-shifts of the excitation energies compared to plain 6-31G calculations in vacuo without changing the essential photophysics. This is a typical picture for extended molecules with delocalized $\pi$-orbitals, in contrast to small molecules of a few-atoms, where large basis sets are necessary.

B. Molecular Aggregates. In section IIIA, we have shown that TDDFT may incorrectly predict excited-state properties for large molecules with donor-acceptor character. Another interesting case, where such problems persist, is molecular aggregates. The separation distances are even larger for aggregates, and the nonlocality should play an even more important role. Modeling of molecular aggregates and assemblies can frequently be reduced to computations on characteristic dimers extracted from the underlying aggregate structure. This allows for the understanding of electronic couplings, ${ }^{33,65}$ excited state, and charge dynamics ${ }^{34}$ seen in ultrafast optical probes. This information in important for light-harvesting and photovoltaic applications of the materials. According to molecular orbital and Hückel theory, every excited state of an isolated molecule should split into a nearly degenerate pair of states for the dimer (the Davydov's pair). The magnitude of splitting characterizes intermolecular coupling. Depending on the orientation of the molecules, 
Table 3. Calculated Vertical Excitation Energies (eV) of the Donor-Acceptor (DA) Compound within Several Levels of Theory ${ }^{a}$

\begin{tabular}{|c|c|c|}
\hline method/basis set & SV geometry & native geometry \\
\hline $\mathrm{HF} / 6-31 \mathrm{G}$ & $4.31(1.59)$ & $4.45(1.56)$ \\
\hline $\mathrm{HF} / 6-31 \mathrm{G}^{*}$ & $4.21(1.57)$ & $4.43(1.48)$ \\
\hline $\mathrm{HF} / 6-31+\mathrm{G}$ & $4.18(1.45)$ & $4.33(1.41)$ \\
\hline $\mathrm{HF} / 6-31+\mathrm{G}^{*}$ & $4.07(1.43)$ & $4.22(1.40)$ \\
\hline HF/6-31G PCM & $4.19(1.70)$ & $4.35(1.60)$ \\
\hline $\mathrm{HF} / 6-31+\mathrm{G}^{*} \mathrm{PCM}$ & $3.96(1.57)$ & $4.11(1.44)$ \\
\hline BHandHLYP/6-31G & $3.71(1.40)$ & $3.66(1.42)$ \\
\hline BHandHLYP/6-31G* & $3.66(1.44)$ & $3.66(1.42)$ \\
\hline BHandHLYP/6-31+G & $3.61(1.33)$ & $3.61(1.33)$ \\
\hline BHandHLYP/6-31+G* & $3.51(1.33)$ & $3.49(1.36)$ \\
\hline BHandHLYP/6-31G PCM & $3.59(1.54)$ & $3.51(1.56)$ \\
\hline BHandHLYP/6-31+G* PCM & $3.42(1.50)$ & $3.45(1.44)$ \\
\hline PBE1PBE/6-31G & $3.12(0.99)$ & $3.04(1.06)$ \\
\hline PBE1PBE/6-31G* & $3.05(0.97)$ & $3.05(1.08)$ \\
\hline PBE1PBE/6-31+G & $3.01(0.99)$ & $2.96(1.03)$ \\
\hline PBE1PBE/6-31+G* & $2.99(1.02)$ & $2.97(1.05)$ \\
\hline PBE1PBE/6-31G PCM & $2.99(1.11)$ & $3.03(1.08)$ \\
\hline PBE1PBE/6-31+G* PCM & $2.87(1.13)$ & $2.91(1.11)$ \\
\hline B3LYP/6-31G & $2.98(0.90)$ & $2.91(0.99)$ \\
\hline B3LYP/6-31G* & $2.95(0.93)$ & $2.93(1.01)$ \\
\hline B3LYP/6-31+G & $2.90(0.89)$ & $2.90(0.98)$ \\
\hline B3LYP/6-31+G* & $2.87(0.91)$ & $2.92(0.99)$ \\
\hline B3LYP/6-31G PCM & $2.85(1.02)$ & $2.77(1.14)$ \\
\hline B3LYP/6-31+G* PCM & $2.73(1.04)$ & $2.75(1.18)$ \\
\hline $\mathrm{BP} 86 / 6-31 \mathrm{G}$ & $2.39(0.62)$ & $2.34(0.72)$ \\
\hline $\mathrm{BP} 86 / 6-31 \mathrm{G}^{*}$ & $2.37(0.62)$ & $2.33(0.71)$ \\
\hline $\mathrm{BP} 86 / 6-31+\mathrm{G}$ & $2.34(0.62)$ & $2.27(0.69)$ \\
\hline $\mathrm{BP} 86 / 6-31+\mathrm{G}^{*}$ & $2.31(0.63)$ & $2.31(0.72)$ \\
\hline BP86/6-31G PCM & $2.25(0.72)$ & $2.20(0.86)$ \\
\hline BP86/6-31+G* PCM & $2.16(0.74)$ & $2.17(0.91)$ \\
\hline
\end{tabular}

${ }^{a}$ The corresponding oscillator strengths $f$ are given in parentheses.

either the lower or higher energy state would acquire a larger oscillator strength. These two classes are classified as J or $\mathrm{H}$ aggregates, respectively. ${ }^{55}$ In this section, we investigate both cases.

First, we examine the thiophene dimers (2Thio4) exemplifying the $\mathrm{H}$-aggregate case. In the lower left-hand corner of Figure 2, we plot the calculated excitation energies for thiophene dimers compared to the single molecule limit. Table 4 shows the respective data. The experimental result is from ref 66. Thiophenes form H-aggregates in crystals. Experimentally, this is exemplified by a blue-shifted absorption and small fluorescence yield compared to the corresponding properties of the monomer. Parallel orientation of the molecules in a dimer results in a positive intermolecular coupling term. This shows up as a higher-lying optical state (in phase combination of monomer's wave functions) than the dark state (out of phase combination of monomer's wave functions). This picture holds relatively well for functionals with a large fraction of orbital exchange (HF and $\mathrm{BHandH}-$ LYP): the Davydov's states split equally up and down from the isolated molecule limit (see Figure 2) and appear as a linear combination of the monomer states in the transition orbitals (see Table 5). With less than $50 \%$ exchange, several spurious charge-transfer states appear. A smaller fraction of exchange generally means a larger number
Table 4. Calculated Excitation Energies (eV) of Thiophene Oligomer (1Th4) and Its Dimer (2Th4)a

\begin{tabular}{clrccc}
\hline molecule & method & $\begin{array}{c}\% \\
\text { exchange }\end{array}$ & $\begin{array}{c}\text { optical } \\
\text { state }\end{array}$ & $\begin{array}{c}\text { dark } \\
\text { state }\end{array}$ & $\begin{array}{c}\text { no. of CT } \\
\text { states }\end{array}$ \\
\hline 1Th4 & HF & 100 & $3.59(1.47)$ & & \\
1Th4 & BHandHLYP & 50 & $3.36(1.28)$ & & \\
1Th4 & PBE1PBE & 25 & $3.14(1.26)$ & & \\
1Th4 & B3LYP & 20 & $3.04(1.22)$ & & \\
1Th4 & TPSS 10\% & 10 & $2.98(1.21)$ & & \\
1Th4 & TPSS & 0 & $2.82(1.13)$ & & \\
1Th4 & BP86 & 0 & $2.77(1.09)$ & & \\
1Th4 & SVWN & 0 & $2.77(1.08)$ & & \\
2Th4 & HF & 100 & $3.67(2.21)$ & 3.47 & 0 \\
2Th4 & BHandHLYP & 50 & $3.45(2.19)$ & 3.21 & 0 \\
2Th4 & PBE1PBE & 25 & $3.24(2.19)$ & 2.97 & 1 \\
2Th4 & B3LYP & 20 & $3.15(1.5)$ & 2.88 & 2 \\
2Th4 & TPSS 10\% & 10 & $3.08(1.35)$ & & 3 \\
2Th4 & TPSS & 0 & $2.92(1.94)$ & & 4 \\
2Th4 & BP86 & 0 & $2.86(1.86)$ & & 4 \\
2Th4 & SVWN & 0 & $2.86(1.83)$ & & 4 \\
\hline
\end{tabular}

${ }^{a}$ The corresponding oscillator strengths $f$ are given in parentheses. Experimental absorption maximum is $\omega_{\mathrm{abs}}=2.7 \mathrm{eV}$ for Th4 in solution.

of charge-transfer states below the first optical singlet. For example, LDA gives at least 4. For the BP86 GGA, the first optical state is given as state 6 instead of 2 . In this case, Figure 2 shows these states as stars. They are heavily mixed with dark and optically allowed excitonic states (e.g., Table 5). We further notice that these spurious charge-transfer states disappear (shift upward in energy) with increasing separation between monomers for hybrid functionals with small amount of orbital exchange (B3LYP and PBE1PBE) but carry on for all LDA and GGA models (not shown). Subsequently, a small fraction of the HF exchange would help to predict the correct monomer limit of excitation energies in the aggregate at large intermolecular separations ( $>5 \AA$ ) when the interchromophore orbital overlap vanishes but fails at smaller distances. In contrast, the CT problems persist for GGA semilocal functionals at all intermolecular separations, which agrees with the results obtained for the ethylene dimer (offsite excitations). ${ }^{67}$ The failure of semilocal functionals to properly describe and characterize these low-lying states means that TDDFT would provide a highly unreliable description of electronic coupling and excitonic effects in such molecular aggregates.

In a second example of molecular assemblies, we consider a form of chlorophyll (Bchl-a) and its dimer (2Bchl-a) from the light-harvesting system of purple bacteria. The computational results are shown in the lower right-hand corner of Figure 2 and Table 6 , and the experimental numbers are from ref 68 . The $\alpha \beta$ Bchls- $a$ pair forms a near J-aggregate complex as evidenced by the red-shifted first optical states in the dimer. In this case, the dark states lie above the optical ones. The full circular LHCII complex has, however, different properties suitable for efficient light-harvesting. ${ }^{35,36,68} \mathrm{We}$ see an interesting trend for the chlorophyll monomers. As more orbital exchange is included, the predicted singlet energy is reduced. This is probably because of a more localized nature of the excited state and compact 2D size of the molecule. This trend may be rationalized by noting that exchange 
Table 5. Transition Orbitals of Thiophene Dimer (2Th4) ${ }^{a}$

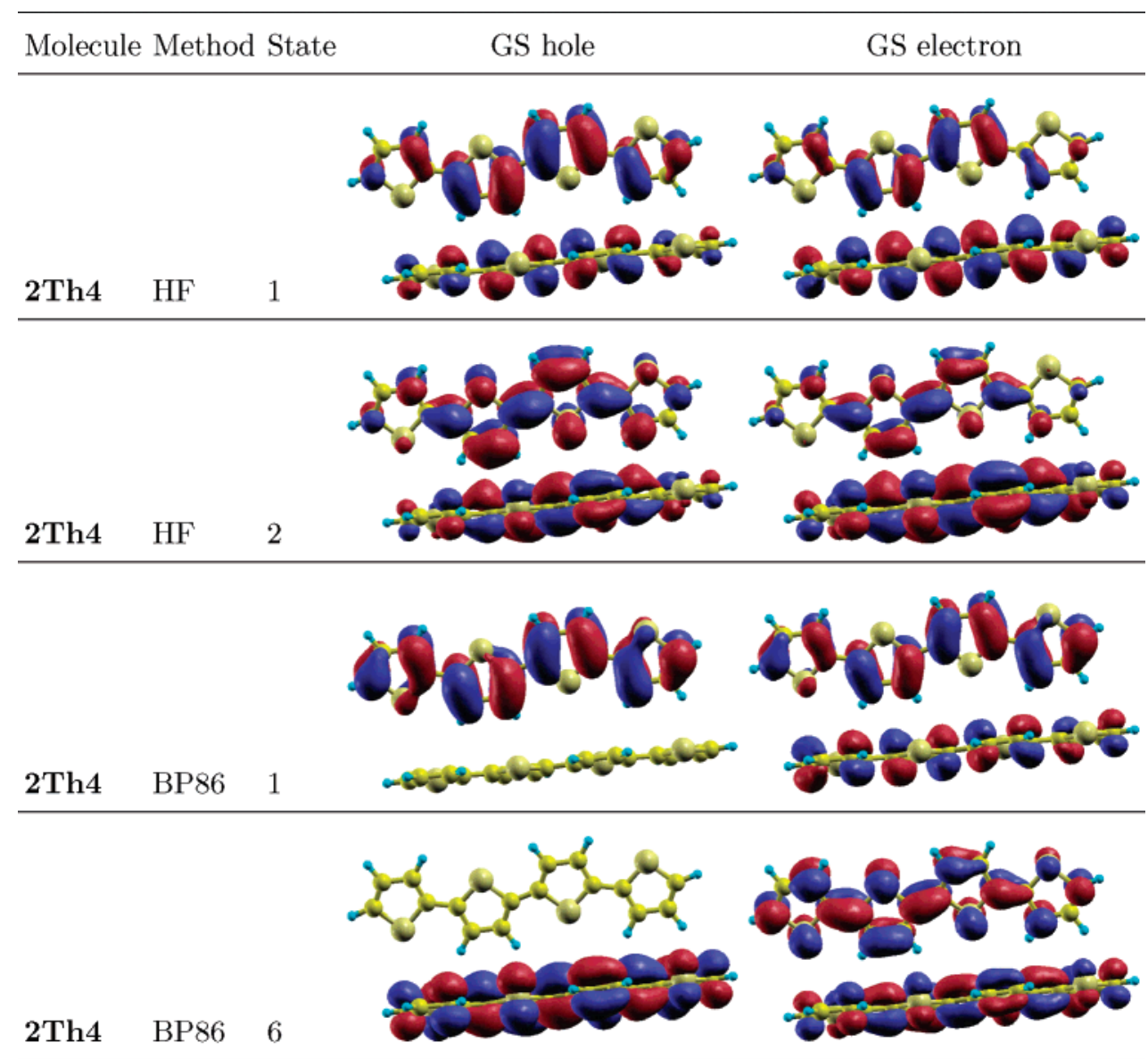

a The top two and bottom two rows represent Hartree-Fock and BP86 limits. States 1 and 2 in the HF limit refer to the dark and optical transitions in the Davydov's pair, respectively. States 1 and 6 in the BP86 limit show charge-transfer character in the lowest dark and optically allowed transitions, respectively.

Table 6. Calculated Excitation Energies (eV) of Bacteriochlorophyll a (1Bchl-a) and $\alpha \beta$ Bchls-a Dimer $(2 \mathrm{Bchl}-\mathrm{a})^{\mathrm{a}}$

\begin{tabular}{llrcrl}
\hline molecule & \multicolumn{1}{c}{ method } & $\begin{array}{c}\text { \% } \\
\text { exchange }\end{array}$ & $\begin{array}{c}\text { optical } \\
\text { state }\end{array}$ & $\begin{array}{c}\text { dark } \\
\text { state }\end{array}$ & $\begin{array}{c}\text { CT } \\
\text { states }\end{array}$ \\
\hline 1Bchl-a & HF & 100 & $1.21(0.42)$ & & \\
1Bchl-a & BHandHLYP & 50 & $1.81(0.40)$ & & \\
1Bchl-a & PBE1PBE & 25 & $1.90(0.36)$ & & \\
1Bchl-a & B3LYP & 20 & $1.89(0.35)$ & & \\
1Bchl-a & TPSS 10\% & 10 & $1.91(0.33)$ & & \\
1Bchl-a & TPSS & 0 & $1.88(0.30)$ & & \\
1Bchl-a & BP86 & 0 & $1.85(0.27)$ & & \\
1Bchl-a & SVWN & 0 & $1.84(0.27)$ & & \\
2Bchl-a & HF & 100 & $1.12(0.90)$ & $1.32(0.08)$ & 0 \\
2Bchl-a & BHandHLYP & 50 & $1.71(0.89)$ & $1.85(0.04)$ & 0 \\
2Bchl-a & PBE1PBE & 25 & $1.90(0.54)$ & & 2 \\
2Bchl-a & B3LYP & 20 & $1.88(0.61)$ & & 2 \\
2Bchl-a & TPSS 10\% & 10 & $1.88(0.67)$ & & 2 \\
2Bchl-a & TPSS & 0 & $1.84(0.64)$ & & 4 \\
2Bchl-a & BP86 & 0 & $1.81(0.62)$ & & 4 \\
2Bchl-a & SWVM & 0 & $1.85(0.64)$ & & 4 \\
\hline
\end{tabular}

${ }^{a}$ The corresponding oscillator strengths $f$ are given in parentheses. The experimental absorption maximum of $Q_{x}$ band of Bchls-a is at about $1.6 \mathrm{eV}$ for a light-harvesting antenna.

creates a steeper optical potential comparable to the core contraction. The net effect would be a smaller optical gap for orbital based functionals and a larger gap for semilocal ones. In the dimer, we observe the expected superposition of the monomer's states in the transition orbitals for HF and BHandHLYP methods. As an example, Table 7 shows the first dominant pair of transition orbitals localized on one chromophore for the first and second excited state in the HF limit. The second pair (not shown) involved the second molecule, respectively. Below 50\% exchange, however, charge-transfer states begin to contaminate the optical spectra (see Table 7) making it impossible to reliably identify the states involved. Formation of such ghost states have been observed before in chlorophylls treated with TDDFT based on GGA models. ${ }^{69,70}$ Finally we note that recently developed functionals based on meta-GGA extensions (TPSS and TPSShybrid) are subject to the same charge-transfer problems as the other approaches for both thiophene and chlorophyll dimers (Tables 4 and 6). The large fraction of the orbital exchange turns out to be the only factor affecting the quality of the results.

Recent TDDFT study of a much smaller system, the ethylene dimer, shows a similar problem with CT states in the symmetric dimers. ${ }^{67,71,72}$ Notably, in our examples of 2Thio4 and 2Bchl-a, the monomers are not identical. Consequently the observed CT states have nonzero net charge transferred between chromophores (see Tables 5 and 7), 
Table 7. Transition Orbitals of $\alpha \beta$ Bacteriochlorophyll a Dimer (2Bchl-a) ${ }^{a}$

Molecule Method State GS hole GS electron

Bchl-a HF 1
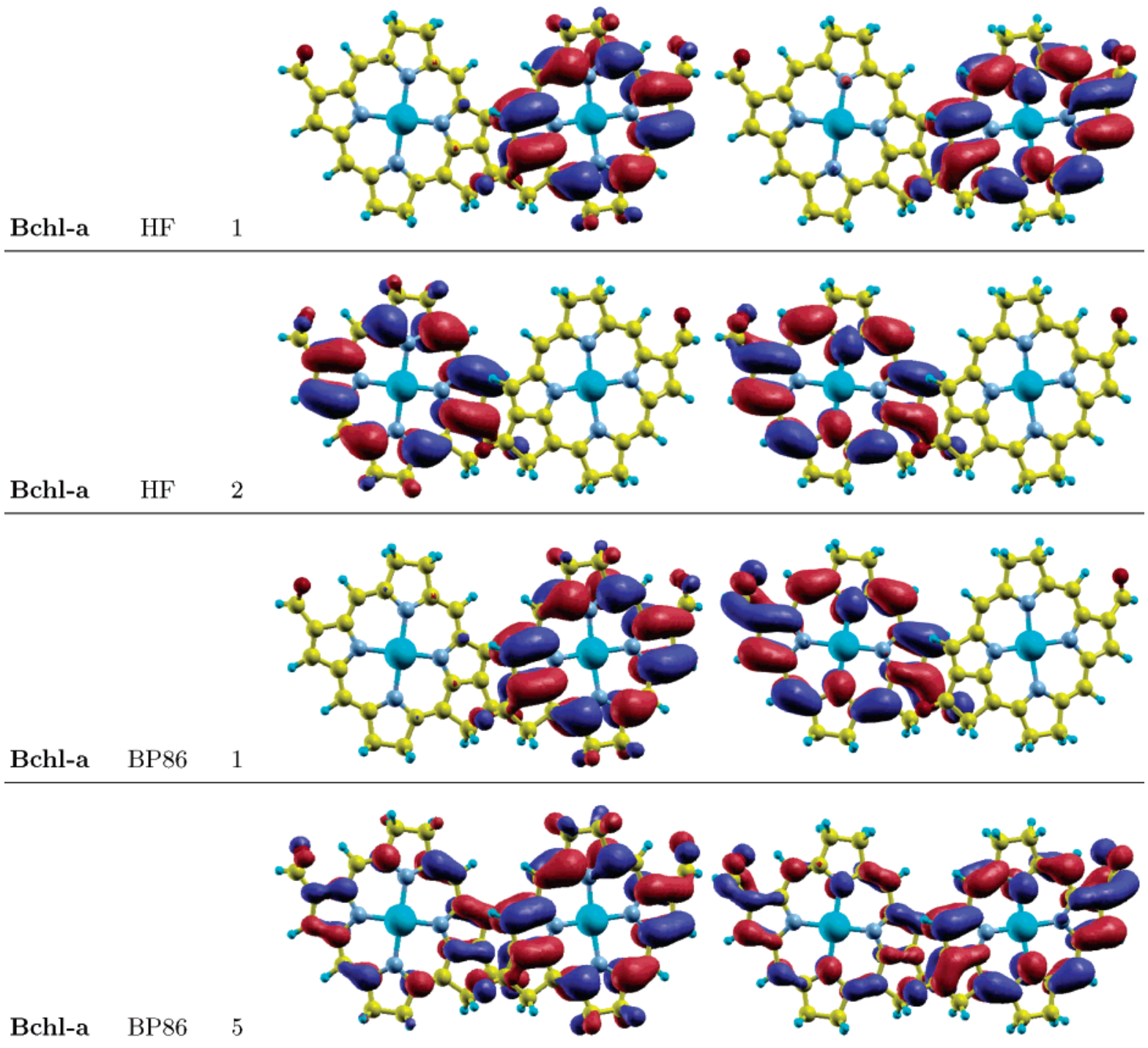

a The top two and bottom two rows represent Hartree-Fock and BP86 limits, respectively. States 1 and 2 in the HF limit refer to the dark and optical transitions in the Davydov's pair, respectively. States 1 and 5 in the BP86 limit show charge-transfer character in the lowest dark and optically allowed transitions, respectively.

opposite to what has been observed in a perfectly symmetric case due to symmetry reasons. ${ }^{67}$ In any case, it is an inaccurate xc-potential that leads to the appearance of unphysical low-lying states, generally dubbed as 'CT states', irrespectively if the actual charge is transferred or not upon excitation. We emphasize that physical analysis of coupling between various molecular regions is very important when deciding whether TD-DFT is applicable to 'problematic' cases or not: Small overlap between individual subsystems of a large system is particularly susceptible to the CT problems. Either vanishing overlap (e.g., large separations between monomers) or 'strong' communication (e.g., fully $\pi$-conjugated bridge) will help to offset the CT failures (i.e., here hybrid functionals with small amount of the orbital exchange may work). For example, the CT problems in donor-acceptor substituted compounds would be even more severe if the donor/acceptor subsystems are separated by some chemical defect such as an $\mathrm{sp}^{3}$ kink. These conclusions fully agree with the general analysis in refs 67, 71, and 72 .

\section{Conclusions}

In this study, we have examined the performance of the TDDFT approach for calculations of excited states. The benchmark systems include several polar donor-acceptor substituted compounds and molecular aggregates. These are typical examples of nanosized molecules currently in the focus of applied quantum chemistry. For all of the systems studied, we have employed a wide range of modern functionals including state-of-the-art meta-GGAs. Overall, today's available density functionals often do not handle chargetransfer states properly. We see that no amount of sophistication in the currently available semilocal functionals describes the CT states successfully. These problems, however, can be overcome by including a larger fraction of orbital exchange, but this is at the expense of accuracy. We point 
out that exact-exchange in density functional theory and Hartree-Fock exchange are not identical, and, in particular, they differ in their representation of virtual orbitals. EXX DFT virtual orbitals are more strongly bound than their HF analogies. This difference means that EXX-based virtual orbitals for the LUMO should be more localized than the $\mathrm{HF}$ analog. Consequently, our results will overemphasize the trends but not create new ones. However, the general conclusions of this work remain valid, and most of the charge-transfer problems persist.

An optimal fraction of orbital exchange is not known, and, most likely, is a system-dependent parameter. Nevertheless, it is possible to formulate several practical approaches based on the trends observed in this study and our previous experience that may overcome the outlined difficulties. We demonstrate that the excited-state geometries from TDDFT calculations using semilocal functionals can be unreliable and susceptible to CT problems. Combining of geometry optimization using functionals with $50 \%$ or more orbital exchange (e.g., HF or BHandHLYP) with excited-state calculations using functionals of lower hybrid content (e.g., B3LYP of PBE1PBE) would prevent drastic geometrical relaxation facilitating formation of ionic states. ${ }^{42}$ Such combinations of different methodologies have been successfully used in the past. For example, coupling of semiempirical AM1 (geometry) and INDO/S (electronic excitations) approaches has worked very well for many molecular systems. ${ }^{34,73}$ Alternatively, using functionals with higher fraction of the orbital exchange (e.g., BHandHLYP) for the entire calculation set would result in a qualitatively correct physical description, but the electronic excitation energies and the respective oscillator strengths would be overestimated. ${ }^{34}$ Similar schemes can be applied to the excited-state description of aggregates and films: the intermolecular couplings can be derived from calculations with a high percentage of the orbital exchange (e.g., HF or BHandHLYP), whereas electronic excitations of a single chromophore can be calculated with numerically accurate DFT workhorses such as B3LYP or PBE1PBE. This would provide a reduced Hamiltonian description to the subsequent modeling. ${ }^{34,73}$ There have been several successful attempts to develop new specific functionals free of CT problems, including asymptotically corrected functionals based on HF exchange LC-TDDFT ${ }^{74,75}$ and CAM-B3LYP' ${ }^{76-78}$ and 'simple correction schemes' ${ }^{\prime}{ }^{79,80}$ The performance of these new functionals is yet to be thoroughly tested for extended molecular systems, and these methods need to be widely adopted in standard computational packages. We should also mention several recent noncanonical DFT-based approaches for electronic excitations, which address the above problems as well and are currently making their way into standard computational packages. This includes techniques based on the DFT-MRCI method, ${ }^{10,69}$ nonlocal density current VK functional, ${ }^{81} \mathrm{GW}$ approximation (GWA), ${ }^{150}$ and Bethe-Salpeter equation (BSE). ${ }^{82-84}$

We hope that these illustrated failures of TDDFT will motivate future work on developing exchange-correlation functionals (and kernels) which can better describe these long-range excited states.
Acknowledgment. This work was carried out under the auspices of the National Nuclear Security Administration of the U.S. Department of Energy at Los Alamos National Laboratory. The research at LANL is supported by the Center for Nonlinear Studies (CNLS) and the LANL LDRD program. This support is gratefully acknowledged.

\section{References}

(1) Gross, E. K. U.; Dobson, J. F.; Petersilka, M. Density Functional Theory; Springer: Berlin, 1996; Vol. 181.

(2) Koch, W.; Holthausen, M. C. A Chemist's Guide to Density Functional Theory; Willey-VCH: Weinheim, 2000.

(3) Casida, M. E. Recent Advances in Density-Functional Methods; World Scientific: Singapore, 1995; Vol. 3 of Part I.

(4) Casida, M. E.; Jamorski, C.; Casida, K. C.; Salahub, D. R. J. Chem. Phys. 1998, 108, 4439.

(5) Furche, F.; Burke, K. Ann. Rep. Comp. Chem. 2005, 1, 19.

(6) Peumans, P.; Uchida, S.; Forrest, S. R. Nature 2003, 425, 158.

(7) Markvart, T. Prog. Quantum Electron. 2000, 24, 107.

(8) Itkis, M. E.; Borondics, F.; Yu, A. P.; Haddon, R. C. Science 2006, 312, 413.

(9) Bartos, F. J. Contr. Eng. 2003, 50, 52.

(10) Grimme, S. Rev. Comp. Chem. 2004, 20, 153.

(11) Dreuw, A.; Head-Gordon, M. Chem. Rev. 2005, 105, 4009.

(12) Hohenberg, P.; Kohn, W. Phys. Rev. 1964, 136, 864.

(13) Runge, E.; Gross, E. K. U. Phys. Rev. Lett. 1984, 52, 997.

(14) Onida, G.; Reining, L.; Rubio, A. Rev. Mod. Phys. 2002, $74,601$.

(15) Rubio, A.; Balbas, L. C.; Alonso, J. A. Phys. Rev. B 1992, $46,4891$.

(16) Serra, L.; Rubio, A. Phys. Rev. Lett. 1997, 78, 1428.

(17) Sahni, V.; Gruenebaum, J.; Perdew, J. P. Phys. Rev. B 1982, 26,4371 .

(18) Langreth, D. C.; Mehl, M. J. Phys. Rev. B 1983, 28, 1809.

(19) Appel, H.; Gross, E. K. U.; Burke, K. Phys. Rev. Lett. 2003, 90, 043005.

(20) Gonze, X.; Scheffler, M. Phys. Rev. Lett. 1999, 82, 4416.

(21) Maitra, N. T.; Zhang, F.; Cave, R. J.; Burke, K. J. Chem. Phys. 2004, 120, 5932.

(22) Maitra, N. T.; Burke, K.; Woodward, C. Phys. Rev. Lett. 2002, 89, 023002.

(23) Tretiak, S.; Chernyak, V. Unpublished.

(24) Grimme, S.; Parac, M. ChemPhysChem 2003, 4, 292.

(25) Dreuw, A.; Head-Gordon, M. J. Am. Chem. Soc. 2004, 126, 4007.

(26) Pogantsch, A.; Heimel, G.; Zojer, E. J. Chem. Phys. 2002, $117,5921$.

(27) Dreuw, A.; Weisman, J. L.; Head-Gordon, M. J. Chem. Phys. 2003, 119, 2943.

(28) Tretiak, S.; Igumenshchev, K.; Chernyak, V. Phys. Rev. B 2005, 71, 33201.

(29) Tozer, D. J. J. Chem. Phys. 2003, 119, 12697. 
(30) Dierksen, M.; Grimme, S. J. Phys. Chem. A 2004, 108, 10225.

(31) Tretiak, S.; Saxena, A.; Martin, R. L.; Bishop, A. R. Phys. Rev. Lett. 2002, 89, 097402.

(32) Spano, F. C. Ann. Rev. Phys. Chem. 2006, 57, 217.

(33) Krueger, B. P.; Scholes, G. D.; Fleming, G. R. J. Phys. Chem. B 1998, 102, 9603.

(34) Bredas, J. L.; Beljonne, D.; Coropceanu, V.; Cornil, J. Chem. Rev. 2004, 104, 4971.

(35) Pullerits, T.; Sundström, V. Acc. Chem. Res. 1996, 29, 381.

(36) Fleming, G. R.; van Grondelle, R. Phys. Today 1994, 47, 48.

(37) Goodson, T. G. Acc. Chem. Res. 2005, 38, 99.

(38) Albota, M.; Beljonne, D.; Brédas, J. L.; Ehrlich, J. E.; Fu, J. Y.; Heikal, A. A.; Hess, S. E.; Kogej, T.; Levin, M. D.; Marder, S. R.; McCord Maughon, D.; Perry, J. W.; Rockel, H.; Rumi, M.; Subramaniam, C.; Webb, W. W.; Wu, X. L.; $\mathrm{Xu}, \mathrm{C}$. Science 1998, 281, 1653.

(39) Dodabalapur, A.; Tousi, L.; Katz, H. E. Science 1995, 268, 270.

(40) Sakaguchi, H.; Matsumura, H.; Gong, H.; Abouelwafa, A. M. Science 2005, 310, 1002.

(41) Blanchard-Desce, M.; Wortmann, R.; Lebus, S.; Lehn, J.; Kramer, P. Chem. Phys. Lett. 1995, 243, 526.

(42) Katan, C.; Terenziani, F.; Mongin, O.; Werts, M. H. V.; Porres, L.; Pons, T.; Mertz, J.; Tretiak, S.; Blanchard-Desce, M. J. Phys. Chem. A 2005, 109, 3024.

(43) van der Horst, J. W.; Bobbert, P. A.; Michels, M. A. J.; Brocks, G.; Kelly, P. J. Phys. Rev. Lett. 1999, 83, 4413.

(44) He, Z.; Sundstrom, V.; Pullerits, T. J. Phys. Chem. B 2002, 106, 11606.

(45) Linnanto, J.; Korppi-Tommola, J. Phys. Chem. Chem. Phys. 2006, 8, 663 .

(46) Ahlrichs, R.; Bar, M.; Haser, M.; Horn, H.; Kolmel, C. Chem. Phys. Lett. 1989, 162, 165.

(47) Furche, F.; Ahlrichs, R. J. Chem. Phys. 2002, 117, 7433.

(48) Masunov, A. M.; Tretiak, S. J. Phys. Chem. B 2004, 108, 899.

(49) Kloc, C.; Laudise, R. A.; Katz, H. E.; Haddon, R. C. Adv. Mat. 1998, 10, 379 .

(50) Koepke, J.; Hu, X. C.; Muenke, C.; Schulten, K.; Michel, H. Structure 1996, 4, 581.

(51) Frisch, M. J.; Trucks, G. W.; Schlegel, H. B.; Scuseria, G. E.; Robb, M. A.; Cheeseman, J. R.; Montgomery, J. A., Jr.; Vreven, T.; Kudin, K. N.; Burant, J. C.; Millam, J. M.; Iyengar, S. S.; Tomasi, J.; Barone, V.; Mennucci, B.; Cossi, M.; Scalmani, G.; Rega, N.; Petersson, G. A.; Nakatsuji, H.; Hada, M.; Ehara, M.; Toyota, K.; Fukuda, R.; Hasegawa, J.; Ishida, M.; Nakajima, T.; Honda, Y.; Kitao, O.; Nakai, H.; Klene, M.; Li, X.; Knox, J. E.; Hratchian, H. P.; Cross, J. B.; Bakken, V.; Adamo, C.; Jaramillo, J.; Gomperts, R.; Stratmann, R. E.; Yazyev, O.; Austin, A. J.; Cammi, R.; Pomelli, C.; Ochterski, J. W.; Ayala, P. Y.; Morokuma, K.; Voth, G. A.; Salvador, P.; Dannenberg, J. J.; Zakrzewski, V. G.; Dapprich, S.; Daniels, A. D.; Strain, M. C.; Farkas, O.; Malick, D. K.; Rabuck, A. D.; Raghavachari, K.;
Foresman, J. B.; Ortiz, J. V.; Cui, Q.; Baboul, A. G.; Clifford, S.; Cioslowski, J.; Stefanov, B. B.; Liu, G.; Liashenko, A.; Piskorz, P.; Komaromi, I.; Martin, R. L.; Fox, D. J.; T. K. Gaussian 03 (Rev. C.02); Gaussian, Inc.: Wallingford, CT, 2003.

(52) Rassolov, V. A.; Ratner, M. A.; Pople, J. A. J. Chem. Phys. 2000, 112, 4014.

(53) Becke, A. D. J. Chem. Phys. 1993, 98, 1372.

(54) Martin, R. L. J. Chem. Phys. 2003, 118, 4775.

(55) Pope, M.; Swenberg, C. E. Electronic Processes in Organic Crystals; Clarendon Press, Oxford University Press: Oxford, New York, 1982.

(56) Charlot, M.; Izard, N.; Mongin, O.; Riehl, D.; BlanchardDesce, M. Chem. Phys. Lett. 2006, 417, 297.

(57) Magyar, R. J.; Fleszar, A.; Gross, E. K. U. Phys. Rev. B 2004, 69, 45111.

(58) Lu, D. Q.; Chen, G. H.; Perry, J. W.; Goddard, W. A. J. Am. Chem. Soc. 1994, 116, 10679.

(59) Moran, A. M.; Kelley, A. M.; Tretiak, S. Chem. Phys. Lett. 2003, 367, 293.

(60) Rappoport, D.; Furche, F. J. Am. Chem. Soc. 2004, 126, 1277.

(61) Cances, E.; Mennucci, B.; Tomasi, J. J. Chem. Phys. 1997, 107,3032 .

(62) Cossi, M.; Barone, V.; Mennucci, B.; Tomasi, J. Chem. Phys. Lett. 1998, 286, 253.

(63) Mennucci, B.; Cammi, R.; Tomasi, J. J. Chem. Phys. 1998, 109, 2798.

(64) Cossi, M.; Barone, V. J. Chem. Phys. 2001, 115, 4708.

(65) Tretiak, S.; Middleton, C.; Chernyak, V.; Mukamel, S. J. Phys. Chem. B 2000, 104, 9540.

(66) Birnbaum, D.; Fichou, D.; Kohler, B. E. J. Chem. Phys. 1992, 96, 165.

(67) Hieringer, W.; Gorling, A. Chem. Phys. Lett. 2006, 419, 557.

(68) Pullerits, T.; Hess, S.; Herek, J. L.; Sundström, V. J. Phys. Chem. B 1997, 101, 10560.

(69) Parusel, A. B. J.; Grimme, S. J. Phys. Chem. B 2000, 104, 5395.

(70) Sundholm, D. Phys. Chem. Chem. Phys. 2000, 2, 2275.

(71) Hieringer, W.; Gorling, A. Chem. Phys. Lett. 2006, 426, 234.

(72) Dreuw, A.; Head-Gordon, M. Chem. Phys. Lett. 2006, 426, 231.

(73) Tretiak, S.; Mukamel, S. Chem. Rev. 2002, 102, 3171.

(74) Chiba, M.; Tsuneda, T.; Hirao, K. J. Chem. Phys. 2006, 124, 144106.

(75) Tawada, Y.; Tsuneda, T.; Yanagisawa, S.; Yanai, T.; Hirao, K. J. Chem. Phys. 2004, 120, 8425.

(76) Peach, M. J. G.; Helgaker, T.; Salek, P.; Keal, T. W.; Lutnaes, O. B.; Tozer, D. J.; Handy, N. C. Phys. Chem. Chem. Phys. 2006, $8,558$.

(77) Yanai, T.; Tew, D. P.; Handy, N. C. Chem. Phys. Lett. 2004, 393, 51.

(78) Rudberg, E.; Salek, P.; Helgaker, T.; Agren, H. J. Chem. Phys. 2005, 123, 184108.

(79) Neugebauer, J.; Gritsenko, O.; Baerends, E. J. J. Chem. Phys. 2006, 124, 214102. 
Spurious Charge-Transfer Excited States in TDDFT

(80) Gritsenko, O.; Baerends, E. J. J. Chem. Phys. 2004, 121, 655.

(81) Vignale, G.; Kohn, W. Phys. Rev. Lett. 1996, 77, 2037.

(82) Ghosez, P.; Gonze, X.; Godby, R. W. Phys. Rev. B 1997, $56,12811$.
J. Chem. Theory Comput., Vol. 3, No. 3, 2007987

(83) Reining, L.; Olevano, V.; Rubio, A.; Onida, G. Phys. Rev. Lett. 2002, 88, 066404.

(84) Sottile, F.; Olevano, V.; Reining, L. Phys. Rev. Lett. 2003, 91, 056402.

CT600282K 